University of Nebraska - Lincoln

DigitalCommons@University of Nebraska - Lincoln

Faculty Publications, Department of Physics and Astronomy

Research Papers in Physics and Astronomy

11-10-2005

Parametrizations and dynamical analysis of angle-integrated cross sections for double photoionization including nondipole effects

Andrei Y. Istomin

University of Nebraska-Lincoln, aistomin2@unl.edu

Anthony F. Starace

University of Nebraska-Lincoln, astarace1@unl.edu

N. L. Manakov

Voronezh State University, Universitetskaya pl. 1, Voronezh, 394006 Russia, manakov@phys.vsu.ru

A. V. Meremianin

Voronezh State University, meremianin@phys.vsu.ru

A. S. Kheifets

Australian National University, a.kheifets@anu.edu.au

See next page for additional authors

Follow this and additional works at: https://digitalcommons.unl.edu/physicsfacpub

Part of the Physics Commons

Istomin, Andrei Y.; Starace, Anthony F.; Manakov, N. L.; Meremianin, A. V.; Kheifets, A. S.; and Bray, Igor, "Parametrizations and dynamical analysis of angle-integrated cross sections for double photoionization including nondipole effects" (2005). Faculty Publications, Department of Physics and Astronomy. 14. https://digitalcommons.unl.edu/physicsfacpub/14

This Article is brought to you for free and open access by the Research Papers in Physics and Astronomy at DigitalCommons@University of Nebraska - Lincoln. It has been accepted for inclusion in Faculty Publications, Department of Physics and Astronomy by an authorized administrator of DigitalCommons@University of Nebraska Lincoln. 


\section{Authors}

Andrei Y. Istomin, Anthony F. Starace, N. L. Manakov, A. V. Meremianin, A. S. Kheifets, and Igor Bray 


\title{
Parametrizations and dynamical analysis of angle-integrated cross sections for double photoionization including nondipole effects
}

\author{
Andrei Y. Istomin and Anthony F. Starace \\ Department of Physics and Astronomy, The University of Nebraska, Lincoln, Nebraska 68588-0111, USA \\ N. L. Manakov and A. V. Meremianin \\ Department of Physics, Voronezh State University, Voronezh 394006, Russia
}

A. S. Kheifets

Research School of Physical Sciences and Engineering, Australian National University, Canberra ACT 0200, Australia

Igor Bray

Centre for Atomic, Molecular, and Surface Physics, School of Mathematical and Physical Sciences, Murdoch University, Perth, 6150 Australia

(Received 18 July 2005; published 11 November 2005)

\begin{abstract}
Similarly to differential cross sections for one-electron photoionization, the doubly differential cross section for double photoionization (DPI) may be conveniently described by four parameters: the singly differential (with respect to energy sharing) cross section $\left(\sigma_{0}\right)$, the dipole asymmetry parameter $(\beta)$, and two nondipole asymmetry parameters $(\gamma$ and $\delta$ ). Here we derive two model-independent representations for these parameters for DPI from a ${ }^{1} S_{0}$ atomic bound state: $(i)$ in terms of one-dimensional integrals of the polarization-invariant DPI amplitudes and (ii) in terms of the exact two-electron reduced matrix elements. For DPI of He at excess energies, $E_{\text {exc }}$, of $100 \mathrm{eV}, 450 \mathrm{eV}$, and $1 \mathrm{keV}$, we present numerical results for the asymmetry parameters within the framework of the convergent close-coupling theory and compare them with results of lowest-order (in the interelectron interaction) perturbation theory (LOPT). The results for $E_{\text {exc }}=1 \mathrm{keV}$ exhibit a nondipole asymmetry that is large enough to be easily measured experimentally. We find excellent agreement between our LOPT results and other theoretical predictions and experimental data for total cross sections and ratios of double to single ionization cross sections for $K$-shell DPI from several multielectron atoms.
\end{abstract}

DOI: 10.1103/PhysRevA.72.052708

PACS number(s): $32.80 . \mathrm{Fb}$

\section{INTRODUCTION}

The process of double photoionization (DPI), especially for the He atom, has been a subject of intense experimental and theoretical studies in atomic physics during the last decade [1-3]. This is because DPI of He represents a prototype of the complete breakup of a three-body atomic system whose fragments interact via long-range Coulomb forces in both the initial (bound) and the final (continuum) states. Understanding the role of electron correlations in this seemingly simple process, involving two electrons, provides insights to the treatment of electron correlations in this and other breakup processes involving many-electron atoms.

The final state following DPI from the initial ${ }^{1} S_{0}$-state involves two photoelectrons whose total (excess) energy is determined by the incident photon energy and the double ionization threshold energy; such a DPI process is thus generally characterized by four different types of cross sections. The triply differential cross section (TDCS), which is differential in the ejection angles of both electrons as well as in their energy sharing, provides the most detailed information on the dynamics of the process by means of the two-electron angular distributions; its calculation and comparison with experiment serves as the most stringent test of theoretical models employed to evaluate the wave functions of the initial and final states. In view of these circumstances, the TDCS for
PDI of He has been analyzed in great detail both theoretically and experimentally (see [1-3] and references therein). Theoretical analyses have established the general parametrizations for the TDCS in terms of polarization-independent amplitudes and scalar products of vectors that enter the DPI process within the electric dipole approximation (EDA) $[1,4,5]$. Similar parametrizations for the TDCS that include the lowest-order nondipole corrections were derived in Refs. [6-8]. Moreover, in both cases the polarization-invariant amplitudes are expressed in terms of Legendre polynomials and exact two-electron reduced matrix elements. These matrix elements are the only quantities that are to be evaluated numerically within a particular dynamical model. Such parametrizations allow one to deduce the symmetry properties and selection rules for the TDCS; they also have proved helpful in analyzing the experimental TDCS data. Dynamical studies of the TDCSs for various photon energies and excess energy sharings, employing particular models for initial and final state wave functions, provide one with a general understanding of the physical mechanisms underlying the DPI process in particular regimes.

There are three other types of DPI cross sections, which are obtained from the TDCS by its integration over the ejection solid angles of one or both electrons and over the energy sharing. Upon integration of the TDCS over the ejection solid angle of one electron, the resulting doubly differential 
cross section (DDCS) has a form that is identical to the differential (in angles) cross section for the one-electron photoeffect and may thus be characterized by the usual asymmetry parameters. Integration over the remaining ejection solid angle yields the singly differential (in energy sharing) cross section (SDCS); integration over energy sharing yields the total cross section. Studies of these cross sections provide a broader picture of the DPI process. Total DPI cross sections and ratios of double to single ionization cross sections for various atoms are of practical interest for plasma physics and astrophysics, where the precise knowledge of these quantities is necessary to characterize radiation propagation in a gaseous medium. Such cross sections have been analyzed both theoretically (see, e.g., Refs. [9-13]) and experimentally [14-18]). Unlike for the TDCS, however, no parametrizations for the nondipole components of the DDCS (or, equivalently, for the corresponding nondipole asymmetry parameters), have been reported in the literature [19]. Also, unlike for the numerical analyses of DPI cross sections in the EDA (in which case a number of numerical methods for accurate calculations of the DPI amplitude has been developed), the existing estimations for the magnitude of nondipole effects at excess energies $E_{\text {exc }}<1 \mathrm{keV}$ are based only on a lowest-order perturbation theory (LOPT) account of interelectron correlations $[6-8,12,13]$.

In the present work, we analyze DPI angle-integrated cross sections as follows. First, in Sec. II, we apply angular momentum techniques similar to those used in Refs. $[4,6,8]$ to derive model-independent representations for the dipole and lowest-order nondipole parameters that characterize the DDCS and the SDCS for DPI from the ${ }^{1} S_{0}$ state at two different levels of detail: (i) in terms of polarizationindependent amplitudes (which may be useful in analyzing experimental data) and (ii) in terms of exact two-electron matrix elements (which may be useful for theoretical calculations). Second, in Sec. III A, we analyze these cross sections for DPI of He quantitatively (including the lowestorder nondipole corrections to the EDA) by means of two methods: (i) the convergent close-coupling (CCC) theory [20], whose EDA predictions for the TDCS are in excellent agreement with existing experiments for $\mathrm{He}$ (see, e.g., Ref. [21]), and (ii) the LOPT, which provides EDA results for the TDCS of reasonable accuracy for low excess energies $[22,23]$ and which has been used recently for analyses of nondipole effects in the TDCS for $E_{\text {exc }} \leqslant 450 \mathrm{eV}$ [6-8]. These results thus allow us: (i) to provide reliable CCC predictions for the dipole and nondipole asymmetry parameters (and thus for the magnitude of nondipole effects at particular excess energies), and (ii) to assess the accuracy of the LOPT predictions for these parameters by comparing them to the CCC results. Third, in Sec. III B, we apply the LOPT approach to calculate the total cross sections and the ratio of double to single ionization cross sections for $K$-shell DPI of multielectron neutral atoms. For this case, our LOPT results are in excellent agreement with available experimental data and confirm the prior predictions of Ref. [13].

\section{THEORY}

The TDCS for DPI from the singlet ${ }^{1} S_{0}$ state, taking into account the lowest-order nondipole corrections, has the following form:

$$
\frac{d^{3} \sigma}{d \Omega_{\mathbf{p}_{1}} d \Omega_{\mathbf{p}_{2}} d E_{1}}=\mathcal{A}\left[\left|A_{d}\right|^{2}+2 \operatorname{Re}\left(A_{d} A_{q}^{*}\right)\right],
$$

where $A_{d}$ and $A_{q}$ are the EDA and electric-quadrupole transition amplitudes, respectively, and where we have neglected the quadrupole-quadrupole term $\left|A_{q}\right|^{2} ; \mathcal{A}=4 \pi^{2} \alpha p_{1} p_{2} / \omega$ is a normalization factor, $\alpha=1 / 137.036$ is the fine structure constant, and atomic units are used throughout the paper. The parametrization of the amplitudes $A_{d}$ and $A_{q}$ in terms of the exact dipole and quadrupole two-electron matrix elements were presented in Refs. [4,6], respectively.

The integration of the dipole-quadrupole TDCS over the ejection angles of one of the electrons yields the dipolequadrupole DDCS. The angular distribution of one electron, described by the DDCS, has a shape that is similar to that of the angle-differential cross section for single photoionization (SPI) that accounts for nondipole effects and that is conventionally characterized by four parameters, as follows:

$$
\frac{d \sigma^{S P I}}{d \Omega_{\mathbf{p}}}=\frac{\sigma_{0}^{S P I}}{4 \pi}\left\{1+\beta^{S P I} P_{2}(|\mathbf{e} \cdot \hat{\mathbf{p}}|)+\left(\delta^{S P I}+\gamma^{S P I}|\mathbf{e} \cdot \hat{\mathbf{p}}|^{2}\right)(\hat{\mathbf{k}} \cdot \hat{\mathbf{p}})\right\},
$$

where $\hat{\mathbf{k}}$ defines the direction of the photon wave vector, $\mathbf{k}[\mathbf{k}=(\omega / c) \hat{\mathbf{k}}], \quad P_{2}(|\mathbf{e} \cdot \hat{\mathbf{p}}|)=\left(3|\mathbf{e} \cdot \hat{\mathbf{p}}|^{2}-1\right) / 2, \sigma_{0}^{S P I}$ is the total SPI cross section, and the parameter $\beta^{S P I}$ characterizes the dipole asymmetry of the cross section, while $\gamma^{S P I}$ and $\delta^{S P I}$ characterize the nondipole asymmetry. Parametrization (2) is valid for any elliptic polarization described by the generally complex photon polarization vector $\mathbf{e}\left[\left(\mathbf{e} \cdot \mathbf{e}^{*}\right)=1\right]$. For linear polarization $\left(\mathbf{e}=\mathbf{e}^{*} \equiv \hat{\boldsymbol{\epsilon}}\right),(\hat{\boldsymbol{\epsilon}} \cdot \hat{\mathbf{p}})=\cos \theta$ and $(\hat{\mathbf{k}} \cdot \hat{\mathbf{p}})=\sin \theta \cos \varphi$, where the angles $\theta$ and $\varphi$ are the spherical angles of the vector $\mathbf{p}$ in the coordinate frame whose $x$ and $z$ axes are directed along the vectors $\mathbf{k}$ and $\hat{\epsilon}$, respectively. For circular polarization, $|\mathbf{e} \cdot \hat{\mathbf{p}}|^{2}=[\hat{\mathbf{k}} \times \hat{\mathbf{p}}]^{2} / 2=(\sin \alpha)^{2} / 2$, where $\alpha$ is the angle between the vectors $\hat{\mathbf{p}}$ and $\hat{\mathbf{k}}$; therefore $P_{2}(|\mathbf{e} \cdot \hat{\mathbf{p}}|)$ is proportional to the Legendre polynomial $P_{2}(\cos \alpha): P_{2}(|\mathbf{e} \cdot \hat{\mathbf{p}}|)=(-1 / 2) P_{2}(\cos \alpha)$. As follows from rotational invariance (cf. Ref. [4]), the dipole-quadrupole DDCS for DPI may be presented in a form that is identical to that in Eq. (2),

$$
\begin{aligned}
\frac{d^{2} \sigma}{d \Omega_{\mathbf{p}_{1}} d E_{1}}= & \frac{\sigma_{0}\left(E_{1}\right)}{4 \pi}\left\{1+\beta\left(E_{1}\right) P_{2}\left(\left|\mathbf{e} \cdot \hat{\mathbf{p}}_{1}\right|\right)\right. \\
& \left.+\left[\delta\left(E_{1}\right)+\gamma\left(E_{1}\right)\left|\mathbf{e} \cdot \hat{\mathbf{p}}_{1}\right|^{2}\right]\left(\hat{\mathbf{k}} \cdot \hat{\mathbf{p}}_{1}\right)\right\},
\end{aligned}
$$

where, however, besides their dependence upon the photon frequency $\omega$, the parameters $\sigma_{0}\left(E_{1}\right), \beta\left(E_{1}\right), \gamma\left(E_{1}\right)$, and $\delta\left(E_{1}\right)$ depend also upon the energy of one of the photoelectrons, $E_{1}$. By integrating Eq. (2) over $\Omega_{\mathrm{p}}$, one obtains the total SPI cross section, $\sigma_{0}^{S P I}$, while by integrating Eq. (3) over $\Omega_{\mathbf{p}_{1}}$, one obtains the DPI SDCS,

$$
\frac{d \sigma}{d E_{1}} \equiv \sigma_{0}\left(E_{1}\right)
$$

The total DPI cross section is then given by [13], 


$$
\sigma^{++}=\int_{0}^{E_{\mathrm{exc}} / 2} \sigma_{0}\left(E_{1}\right) d E_{1},
$$

where $E_{\text {exc }}=\omega-I^{++}$is the excess energy and $I^{++}$is the double ionization threshold energy.

The parametrizations of the dipole and nondipole coefficients for SPI in Eq. (2) in terms of the exact matrix elements are well known. By using the parametrizations for the DPI amplitudes and the TDCS given in Refs. [4,6] and the techniques developed in Ref. [4], it is possible to derive $a b$ initio parametrizations for the coefficients $\sigma_{0}\left(E_{1}\right), \beta\left(E_{1}\right), \gamma\left(E_{1}\right)$, and $\delta\left(E_{1}\right)$ in Eq. (3) in terms of (i) polarization-invariant amplitudes and (ii) exact two-electron reduced matrix elements. These two parametrizations are derived below.

\section{A. DPI transition amplitude}

For DPI from a ${ }^{1} S_{0}$-state $|0\rangle$, the transition amplitude $A$ taking into account nondipole effects to lowest order involves dipole and quadrupole components and has the following form [8]:

$$
A=A_{d}+A_{q}=\left\langle\mathbf{p}_{1} \mathbf{p}_{2}\left|(\mathbf{e} \cdot \mathbf{D})+\left(\{\hat{\mathbf{k}} \otimes \mathbf{e}\}_{2} \cdot Q_{2}\right)\right| 0\right\rangle .
$$

In the velocity gauge, $\mathbf{D}=-i\left(\nabla_{1}+\nabla_{2}\right)$ and $Q_{2 m}=\alpha \omega\left(\left\{\mathbf{r}_{1}\right.\right.$ $\left.\otimes \nabla_{1}\right\}_{2 m}+\left\{\mathbf{r}_{2} \otimes \nabla_{2}\right\}_{2 m}$ ). (Standard notations [24] for irreducible tensor products, $6 j$ symbols, etc., are used.)

The parametrizations for the amplitudes $A_{d}$ and $A_{q}$ as well as for the TDCS in Eq. (1) are derived using the following expansion of the two-electron final state $\left|\mathbf{p}_{1} \mathbf{p}_{2}\right\rangle$ in terms of modified bipolar harmonics, $\mathcal{C}_{l m}^{l_{1} l_{2} *}\left(\hat{\mathbf{p}}_{1}, \hat{\mathbf{p}}_{2}\right)$ :

$$
\left|\mathbf{p}_{1} \mathbf{p}_{2}\right\rangle=\sum_{l_{1} l_{2} l m} \mathcal{C}_{l m}^{l_{1} l_{2} *}\left(\hat{\mathbf{p}}_{1}, \hat{\mathbf{p}}_{2}\right)\left|p_{1} p_{2} ;\left(l_{1} l_{2}\right) l m\right\rangle,
$$

where

$$
\begin{aligned}
\mathcal{C}_{L M}^{l l^{\prime}}\left(\hat{\mathbf{r}}, \hat{\mathbf{r}}^{\prime}\right) & =\sum_{m, m^{\prime}} C_{l m l^{\prime} m^{\prime}}^{L M} C_{l m}(\hat{\mathbf{r}}) C_{l^{\prime} m^{\prime}}\left(\hat{\mathbf{r}}^{\prime}\right) \\
& \equiv\left\{C_{l m}(\hat{\mathbf{r}}) \otimes C_{l^{\prime} m^{\prime}}\left(\hat{\mathbf{r}}^{\prime}\right)\right\}_{L M},
\end{aligned}
$$

$C_{l m}(\hat{\mathbf{r}}) \equiv \sqrt{4 \pi /(2 l+1)} Y_{l m}(\hat{\mathbf{r}})$ is a modified spherical harmonic [24], and $C_{l m l^{\prime} m^{\prime}}^{L M}$ is a Clebsch-Gordon coefficient. With these definitions, the dipole $\left(A_{d}\right)$ and quadrupole $\left(A_{q}\right)$ components of the DPI transition amplitude are given by $[4,6]$,

$$
\begin{gathered}
A_{d}=\frac{1}{\sqrt{3}} \sum_{l_{1}, l_{2}=0}^{\infty} d_{l_{1} l_{2}}\left(p_{1}, p_{2}\right)\left(\mathbf{e} \cdot \mathcal{C}_{1}^{l_{1}, l_{2}}\left(\hat{\mathbf{p}}_{1}, \hat{\mathbf{p}}_{2}\right)\right), \\
A_{q}=\frac{1}{\sqrt{5}} \sum_{l_{1}, l_{2}=0}^{\infty} q_{l_{1} l_{2}}\left(p_{1}, p_{2}\right)\left(\{\mathbf{e} \otimes \hat{\mathbf{k}}\}_{2} \cdot \mathcal{C}_{2}^{l_{1}, l_{2}}\left(\hat{\mathbf{p}}_{1}, \hat{\mathbf{p}}_{2}\right)\right),
\end{gathered}
$$

where

$$
\begin{aligned}
& d_{l l^{\prime}}\left(p, p^{\prime}\right) \equiv\left\langle p p^{\prime} ;\left(l l^{\prime}\right) 1\|\mathbf{D}\| 0\right\rangle, \\
& q_{l l^{\prime}}\left(p, p^{\prime}\right) \equiv\left\langle p p^{\prime} ;\left(l l^{\prime}\right) 2\left\|Q_{2}\right\| 0\right\rangle
\end{aligned}
$$

are the reduced matrix elements of the electric-dipole and electric-quadrupole operators between the ${ }^{1} S_{0}$-state and the
$P$-wave and $D$-wave components of the two-electron continuum state $\left|\mathbf{p} \mathbf{p}^{\prime}\right\rangle$ having individual photoelectron angular momenta $l$ and $l^{\prime}$, respectively. The reduced matrix elements are defined by the Wigner-Eckart theorem [24], taking into account Eq. (7). Note that only $d_{l_{1} l_{2}}\left(p_{1}, p_{2}\right)$ with $l_{2}=l_{1} \pm 1$ are nonzero in the dipole amplitude, while only $q_{l_{1} l_{2}}\left(p_{1}, p_{2}\right)$ with $l_{2}=l_{1}, l_{1} \pm 2$ are nonzero in the quadrupole amplitude.

Eqs. (9) and (10) reduce to the following modelindependent parametrizations for $A_{d}$ and $A_{q}[4,6]$ :

$$
A_{d}=f_{1}\left(\mathbf{e} \cdot \hat{\mathbf{p}}_{1}\right)+f_{2}\left(\mathbf{e} \cdot \hat{\mathbf{p}}_{2}\right),
$$

$$
\begin{aligned}
A_{q}= & g_{1}\left(\mathbf{e} \cdot \hat{\mathbf{p}}_{1}\right)\left(\hat{\mathbf{p}}_{1} \cdot \hat{\mathbf{k}}\right)+g_{2}\left(\mathbf{e} \cdot \hat{\mathbf{p}}_{2}\right)\left(\hat{\mathbf{p}}_{2} \cdot \hat{\mathbf{k}}\right)+g_{S}\left[\left(\mathbf{e} \cdot \hat{\mathbf{p}}_{1}\right)\left(\hat{\mathbf{p}}_{2} \cdot \hat{\mathbf{k}}\right)\right. \\
& \left.+\left(\mathbf{e} \cdot \hat{\mathbf{p}}_{2}\right)\left(\hat{\mathbf{p}}_{1} \cdot \hat{\mathbf{k}}\right)\right],
\end{aligned}
$$

where the polarization-invariant amplitudes $f_{1,2}, g_{1,2}$, and $g_{s}$ depend upon the photoelectron momenta, $p_{1}$ and $p_{2}$, and upon $\cos \theta \equiv\left(\hat{\mathbf{p}}_{1} \cdot \hat{\mathbf{p}}_{2}\right)$, where $\theta$ is their mutual ejection angle. Here we use the following notations: $f_{1} \equiv f\left(p_{1}, p_{2}, \cos \theta\right)$, $f_{2} \equiv f\left(p_{2}, p_{1}, \cos \theta\right), \quad g_{1} \equiv g\left(p_{1}, p_{2}, \cos \theta\right), \quad$ and $\quad g_{2}$ $\equiv g\left(p_{2}, p_{1}, \cos \theta\right)$, where

$$
f\left(p, p^{\prime}, \cos \theta\right)=\sum_{l=1}^{\infty}(-1)^{l+1}\left[\sum_{l^{\prime}=l \pm 1} D_{l l^{\prime}}\left(p, p^{\prime}\right)\right] P_{l}^{(1)}(\cos \theta),
$$

$$
\begin{aligned}
& g_{s}\left(p, p^{\prime}, \cos \theta\right)=\sum_{l=1}^{\infty}(-1)^{l+1}\left[\sum_{l^{\prime}=l \pm 2} Q_{l l^{\prime}}\left(p, p^{\prime}\right) P_{\left(l+l^{\prime} / 2\right)}^{(2)}(\cos \theta)\right. \\
& \left.\quad+\sqrt{6} Q_{l l}\left(p, p^{\prime}\right)\left(P_{l+1}^{(2)}(\cos \theta)-\frac{2 l+3}{2} P_{l}^{(1)}(\cos \theta)\right)\right]
\end{aligned}
$$

$$
\begin{aligned}
& g\left(p, p^{\prime}, \cos \theta\right) \\
& \quad=\sum_{l=2}^{\infty}(-1)^{l}\left[\sum_{l^{\prime}=l \pm 2} Q_{l l^{\prime}}\left(p, p^{\prime}\right)+\sqrt{6} Q_{l l}\left(p, p^{\prime}\right)\right] P_{l}^{(2)}(\cos \theta),
\end{aligned}
$$

and where $P_{l}^{(n)}(x)$ is the $n$th derivative of the Legendre polynomial $P_{l}(x), P_{l}^{(n)}(x)=\left(d^{n} / d x^{n}\right) P_{l}(x)$. The dynamical factors $D_{l_{1} l_{2}}\left(p_{1}, p_{2}\right)$ and $Q_{l_{1} l_{2}}\left(p_{1}, p_{2}\right)$ are defined as follows:

$$
\begin{gathered}
D_{l l^{\prime}}\left(p, p^{\prime}\right)=\frac{d_{l l^{\prime}}\left(p, p^{\prime}\right)}{\sqrt{(2 l+1)\left(2 l^{\prime}+1\right) \max \left(l, l^{\prime}\right)}}, \\
Q_{l l^{\prime}}\left(p, p^{\prime}\right)=\sqrt{\frac{4\left(l+l^{\prime}-2\right) !}{\left(l+l^{\prime}+3\right) !}} q_{l l^{\prime}}\left(p, p^{\prime}\right) .
\end{gathered}
$$

\section{B. Parametrization of the DDCS and SDCS in terms of the polarization-invariant amplitudes}

The formulae for the DPI transition amplitude given above allow one to derive model-independent parametrizations for the DDCS parameters $\sigma_{0}, \beta, \gamma$, and $\delta$ in Eq. (3) in 
terms of the polarization-invariant amplitudes $f_{1,2}(\cos \theta)$, $g_{1,2}(\cos \theta)$, and $g_{s}(\cos \theta)$. The integration of the TDCS in Eq. (1) over $\Omega_{\mathbf{p}_{2}}$ reduces to evaluation of the following integrals:

$$
\begin{gathered}
I_{1}=\int d \Omega_{\mathbf{p}_{2}}\left(\mathbf{a} \cdot \hat{\mathbf{p}}_{2}\right) u_{1}(\cos \theta), \\
I_{2}=\int d \Omega_{\mathbf{p}_{2}}\left(\mathbf{a} \cdot \hat{\mathbf{p}}_{2}\right)\left(\mathbf{b} \cdot \hat{\mathbf{p}}_{2}\right) u_{2}(\cos \theta), \\
I_{3}=\int d \Omega_{\mathbf{p}_{2}}\left(\mathbf{a} \cdot \hat{\mathbf{p}}_{2}\right)\left(\mathbf{b} \cdot \hat{\mathbf{p}}_{2}\right)\left(\mathbf{c} \cdot \hat{\mathbf{p}}_{2}\right) u_{3}(\cos \theta),
\end{gathered}
$$

where the functions $u_{1,2,3}$ involve combinations of the polarization-invariant amplitudes that depend upon $\cos \theta$. These integrals are evaluated in Appendix A. The resulting explicit expressions for the coefficients $\sigma_{0}, \beta, \gamma$, and $\delta$ in Eq. (3) are

$$
\begin{gathered}
\sigma_{0}=\mathcal{A} \frac{8 \pi^{2}}{3} \int_{-1}^{1}\left[\left|f_{1}\right|^{2}+\left|f_{2}\right|^{2}+2 \operatorname{Re}\left(f_{1} f_{2}^{*}\right) x\right] d x, \\
\beta=2-\mathcal{A} \frac{8 \pi^{2}}{\sigma_{0}} \int_{-1}^{1}\left|f_{2}\right|^{2}\left(1-x^{2}\right) d x, \\
\gamma=\mathcal{A} \frac{(4 \pi)^{2}}{\sigma_{0}} \int_{-1}^{1} \operatorname{Re}\left\{f_{1}^{*}\left[g_{1}+g_{2} P_{2}(x)+2 g_{s} x\right]\right. \\
\left.+f_{2}^{*}\left[g_{1} x+g_{2} P_{3}(x)+2 g_{s} P_{2}(x)\right]\right\} d x, \\
\delta= \\
\mathcal{A} \frac{8 \pi^{2}}{\sigma_{0}} \int_{-1}^{1} \operatorname{Re}\left[f_{2}\left(g_{s}^{*}+g_{2}^{*} x\right)\right]\left(1-x^{2}\right) d x,
\end{gathered}
$$

where the polarization-invariant amplitudes $f_{1,2}(x), g_{1,2}(x)$, and $g_{s}(x)$ depend upon the integration variable $x \equiv \cos \theta$. It is interesting to note that the EDA amplitude $f_{1}$ does not contribute to the dipole asymmetry parameter $\beta$ and that neither $f_{1}$ nor the quadrupole amplitude $g_{1}$ contribute to the nondipole asymmetry parameter $\delta$. [This "asymmetry" of Eqs. (19)-(21) with respect to $f_{1}$ and $f_{2}$ (as well as $g_{1}$ and $g_{2}$ ) originates from the fact that the DDCS that Eqs. (18)-(21) describe is defined as an integral of the TDCS over the ejection angles of the electron having momentum $\mathbf{p}_{2}$.] The expressions above give parametrizations for $\sigma_{0}, \beta, \gamma$, and $\delta$ that are independent of the approach used to calculate the polarization-invariant amplitudes.

\section{Parametrization of the DDCS and SDCS in terms of the two-electron matrix elements}

Parametrization of the quantities $\sigma_{0}, \beta, \gamma$, and $\delta$ in terms of the reduced matrix elements in Eq. (11) may be obtained by direct substitution of the expressions for the polarizationinvariant amplitudes [cf. Eqs. (14) and (15) and the immediately preceding text] into Eqs. (18)-(21) followed by evaluation of the multiple integrals involving combinations of
Legendre polynomials. A more convenient alternative, however, is to perform the integration of the TDCS over the ejection angles of one electron $\Omega_{\mathbf{p}_{2}} \equiv\left(\theta_{2}, \varphi_{2}\right)$ by integrating Eq. (1), where the amplitudes $A_{d}$ and $A_{q}$ are given by Eqs. (9) and (10).

The integration of the dipole-dipole term $\left|A_{d}\right|^{2}$ in Eq. (1) may be performed as follows. First, one expands all tensor products in terms of tensor spherical components and performs the integration over $\Omega_{\mathbf{p}_{2}}$ of the two spherical functions that depend upon $\hat{\mathbf{p}}_{2}$. Then, one presents the result as a sum of scalar products of tensors having the form $\left(\left\{\mathbf{e} \otimes C_{l_{1}}\left(\hat{\mathbf{p}}_{1}\right)\right\}_{l_{2}} \cdot\left\{\mathbf{e}^{*} \otimes C_{l_{1}^{\prime}}\left(\hat{\mathbf{p}}_{1}\right)\right\}_{l_{2}}\right)$, where $l_{1}, l_{1}^{\prime}=l_{2} \pm 1$. After recoupling, the result is formally presented as a sum of scalar products $\left(\left\{\mathbf{e} \otimes \mathbf{e}^{*}\right\}_{g} \cdot \mathcal{C}_{g}^{l_{1}, l_{1}^{\prime}}\left(\hat{\mathbf{p}}_{1}, \hat{\mathbf{p}}_{1}\right)\right)$, where $g=0$, 2. Finally, each bipolar harmonic that depends upon two equal arguments is expressed in terms of a single spherical harmonic. The result of the integration of the dipole-dipole term is thus

$$
\begin{aligned}
\int d \Omega_{\mathbf{p}_{2}} A_{d} A_{d}^{*}= & 4 \pi \sum_{g=0,2}\left(\left\{\mathbf{e} \otimes \mathbf{e}^{*}\right\}_{g} \cdot C_{g}\left(\hat{\mathbf{p}}_{1}\right)\right) \\
& \times \sum_{\substack{l_{2}=0 \\
l_{l_{1}=l_{2} \pm 1}}} \frac{(-1)^{l_{2}}}{2 l_{2}+1} C_{l_{1} 0 l_{1}^{\prime} 0}^{g 0}\left\{\begin{array}{ccc}
1 & l_{1}^{\prime} & l_{2} \\
l_{1} & 1 & g
\end{array}\right\} \\
& \times d_{l_{1} l_{2}} d_{l_{1}^{\prime} l_{2}}^{*} \cdot
\end{aligned}
$$

The integration of the dipole-quadrupole term $A_{d} A_{q}^{*}$ in Eq. (1) may be performed similarly and yields

$$
\begin{aligned}
\int d \Omega_{\mathbf{p}_{2}} A_{d} A_{q}^{*}= & 4 \pi \sum_{g=1,3}\left(\left\{\{\mathbf{e} \otimes \hat{\mathbf{k}}\}_{2} \otimes \mathbf{e}\right\}_{g} \cdot C_{g}\left(\hat{\mathbf{p}}_{1}\right)\right) \\
& \times \sum_{l_{2}=2}^{\infty} \sum_{\substack{l_{1}=l_{2} \pm 1 \\
l_{1}^{\prime}=l_{2}, l_{2} \pm 2}} \frac{(-1)^{l_{2}}}{2 l_{2}+1} C_{l_{1} 0 l_{1}^{\prime} 0}^{g 0}\left\{\begin{array}{ccc}
2 & l_{1}^{\prime} & l_{2} \\
l_{1} & 1 & g
\end{array}\right\} \\
& \times d_{l_{1} l_{2}} q_{l_{1}^{\prime} l_{2}}^{*} .
\end{aligned}
$$

After rewriting the scalar products of the tensors involving the vectors $\mathbf{e}$ and $\hat{\mathbf{k}}$ with the spherical harmonic $C_{g}\left(\hat{\mathbf{p}}_{1}\right)$ in Eqs. (22) and (23) in terms of the scalar products $\left(\mathbf{e} \cdot \hat{\mathbf{p}}_{1}\right)$ and $\left(\hat{\mathbf{k}} \cdot \hat{\mathbf{p}}_{1}\right)$, the parametrizations for the coefficients $\sigma_{0}, \beta, \gamma$, and $\delta$ in Eq. (3) may readily be obtained as follows:

$$
\begin{gathered}
\sigma_{0}=(4 \pi)^{2} \frac{\mathcal{A}}{3} \sum_{l_{2}=0}^{\infty} \sum_{l_{1}=l_{2} \pm 1} \frac{\left|d_{l_{1} l_{2}}\right|^{2}}{\left(2 l_{1}+1\right)\left(2 l_{2}+1\right)}, \\
\beta=\frac{(4 \pi)^{2}}{\sigma_{0}} \mathcal{A} \sqrt{\frac{2}{3} \sum_{l_{2}=0}^{\infty} \sum_{l_{1}=l_{2} \pm 1} \frac{(-1)^{l_{2}}}{2 l_{2}+1}} \\
\times C_{l_{1} 0 l_{1}^{\prime} 0}^{20}\left\{\begin{array}{ccc}
1 & l_{1}^{\prime} & l_{2} \\
l_{1}=l_{2} \pm 1 & 1 & 2
\end{array}\right\} d_{l_{1} l_{2}} d_{l_{1}^{\prime} l_{2}}^{*},
\end{gathered}
$$




$$
\begin{gathered}
\gamma=-\frac{(4 \pi)^{2}}{\sigma_{0}} \sqrt{10} \mathcal{A} \sum_{l_{2}=2}^{\infty} \sum_{\substack{l_{1}=l_{2} \pm 1 \\
l_{1}^{\prime}=l_{2}, l_{2} \pm 2}} \frac{(-1)^{l_{2}}}{2 l_{2}+1} \\
\times C_{l_{1} 0 l_{1}^{\prime} 0}^{30}\left\{\begin{array}{ccc}
2 & l_{1}^{\prime} & l_{2} \\
l_{1} & 1 & 3
\end{array}\right\} \operatorname{Re}\left(d_{l_{1} l_{2}} q_{l_{1}^{\prime} l_{2}}^{*}\right), \\
\delta=\frac{(4 \pi)^{2}}{\sigma_{0}} \frac{\mathcal{A}}{\sqrt{5}} \sum_{l_{2}=2}^{\infty} \sum_{l_{1}=l_{2} \pm 1}^{\infty} \frac{(-1)^{l_{2}}}{2 l_{2}+1} \operatorname{Re}\left(d_{l_{1} l_{2}} q_{l_{1}^{\prime} l_{2}}^{*}\right) \\
\times\left(\sqrt{3} C_{l_{1} 0 l_{1}^{\prime} 0}^{10}\left\{\begin{array}{ccc}
l_{1}^{\prime}=l_{2}, l_{2} \pm 2 \\
2 & l_{1}^{\prime} & l_{2} \\
l_{1} & 1 & 1
\end{array}\right\}+\sqrt{2} C_{l_{1} 0 l_{1}^{\prime} 0}^{30}\left\{\begin{array}{lll}
2 & l_{1}^{\prime} & l_{2} \\
l_{1} & 1 & 3
\end{array}\right\}\right) .
\end{gathered}
$$

One sees that dipole-quadrupole terms, which are of the order of $\omega / c$, do not appear in the SDCS given by the parameter $\sigma_{0}$ in Eqs. (18) and (24), i.e., the lowest-order nondipole corrections that contribute to the SDCS are the quadrupole-quadrupole and dipole-octupole terms, which are of the order $(\omega / c)^{2}$ (and hence are not considered here). Also, it is interesting to note that the parameter $\delta$ is generally nonzero. This is in contrast to SPI, for which $\delta^{S P I}$ vanishes for ionization from atomic $s$ subshells [25].

\section{NUMERICAL RESULTS}

\section{A. Double ionization of $\mathrm{He}$}

In this section, we present numerical CCC and LOPT results for DDCSs and SDCSs for DPI of He at three excess energies, $100 \mathrm{eV}, 450 \mathrm{eV}$, and $921 \mathrm{eV}$. The LOPT results presented are gauge invariant as they include both final state (FS) and ground state (GS) correlations to lowest (first) order in $1 / Z$ and use a single basis set of Coulomb orbitals with $Z=2$. The technical details of the calculation of the dipole and quadrupole reduced matrix elements within the LOPT approach are given in Refs. [23,8], respectively. The CCC results are gauge invariant as well, as they effectively include FS and GS correlations to all orders (in the ground state, by using a 20-term Hylleraas expansion for the ground-state wave function, and in the final state, by means of a convergent close-coupling expansion for the two-electron continuum [11]). The reduced matrix elements of the electricquadrupole operator have been extracted from the $(e, 3 e)$ CCC calculation described in Ref. [26] (see Appendix B for details). In Eqs. (24)-(27), the reduced matrix elements $d_{l_{1} l_{2}}$ and $q_{l_{1} l_{2}}$ with $l_{2}$ up to $l_{2}=6$ have been included in both the $\mathrm{CCC}$ and LOPT calculations.

\section{Doubly differential cross sections}

Here we present numerical results for the DDCSs. It is assumed that one of the electrons is detected in the plane spanned by the vectors $\mathbf{e}$ and $\mathbf{k}$. The results for $100 \mathrm{eV}$ and $450 \mathrm{eV}$ are presented for those energy sharings for which experimental measurements of DDCSs and theoretical EDA predictions exist in the literature, i.e., $(99 \mathrm{eV}+1 \mathrm{eV})[27,28]$,
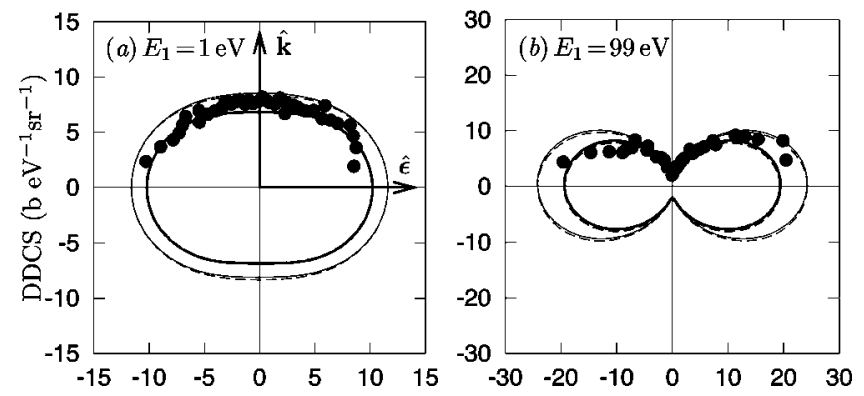

FIG. 1. DDCS for DPI of He for an excess energy of $100 \mathrm{eV}$, linear polarization, and coplanar geometry. Directions of the photon wave vector $\hat{\mathbf{k}}$ and polarization $\hat{\boldsymbol{\epsilon}}$ are as shown in (a). The angular distributions for the electron having energy $E_{1}$ are shown. Full and dashed curves in bold: CCC; full and dashed curves of regular thickness: LOPT. Full curves: dipole-quadrupole results; dashed curves: dipole results. Experimental results [27] are normalized to the CCC results.

$(448 \mathrm{eV}+2 \mathrm{eV})[28,29]$, and $(420 \mathrm{eV}+30 \mathrm{eV})[28,29]$. We note that lowest-order nondipole corrections have been included by means of the asymmetry parameters $\gamma$ and $\delta$ in Eqs. (26) and (27).

Figure 1 shows results for the dipole and dipolequadrupole DDCS for $E_{\mathrm{exc}}=100 \mathrm{eV}$ calculated by the CCC and LOPT approaches, in comparison with the experimental data [27] (which are normalized to the CCC results). One sees that the nondipole forward-backward asymmetries at this excess energy are barely noticeable, and that the predictions of both the CCC and LOPT approaches are in reasonable agreement with each other and with the experimental data.

In Fig. 2, we present the DDCS for an excess energy of $450 \mathrm{eV}$, which is the highest one for which DDCS experimental data are available. One sees that the nondipole asymmetries for this case are more pronounced than for $100 \mathrm{eV}$, particularly for angular distributions of the fast electron. For extremely asymmetric energy sharing [see Figs. 2(a) and 2(b)], we find reasonable agreement of LOPT predictions with both the CCC results and normalized experimental data [29], although the LOPT-calculated cross sections are somewhat larger than the CCC results. For less asymmetric energy sharings [see Figs. 2(c) and 2(d)], the LOPT results overestimate the absolute magnitude of the DDCSs even more. However, the nondipole asymmetries predicted by the CCC and LOPT approaches are in good qualitative agreement.

To illustrate the importance of nondipole effects in photoelectron angular distributions at higher photon energies, we present DDCS predictions for an excess energy of $1 \mathrm{keV}$ (i.e., for a photon energy of $1.079 \mathrm{keV}$ ) in Fig. 3. The energy ratios, $R=E_{2} / E_{1}$, that we have chosen are similar to those in Fig. 2. As expected, one sees that for an excess energy of 1 $\mathrm{keV}$, the nondipole asymmetries in the angular distributions of the fast electron become more significant than for $450 \mathrm{eV}$ and should be observable in experiments.

The DDCSs in Figs. 1-3 have been calculated using Eq. (3), where the parameters $\sigma_{0}, \beta, \gamma$, and $\delta$ have been evaluated by the CCC and LOPT approaches. These parameters are summarized in Table I and allow one to formulate some 

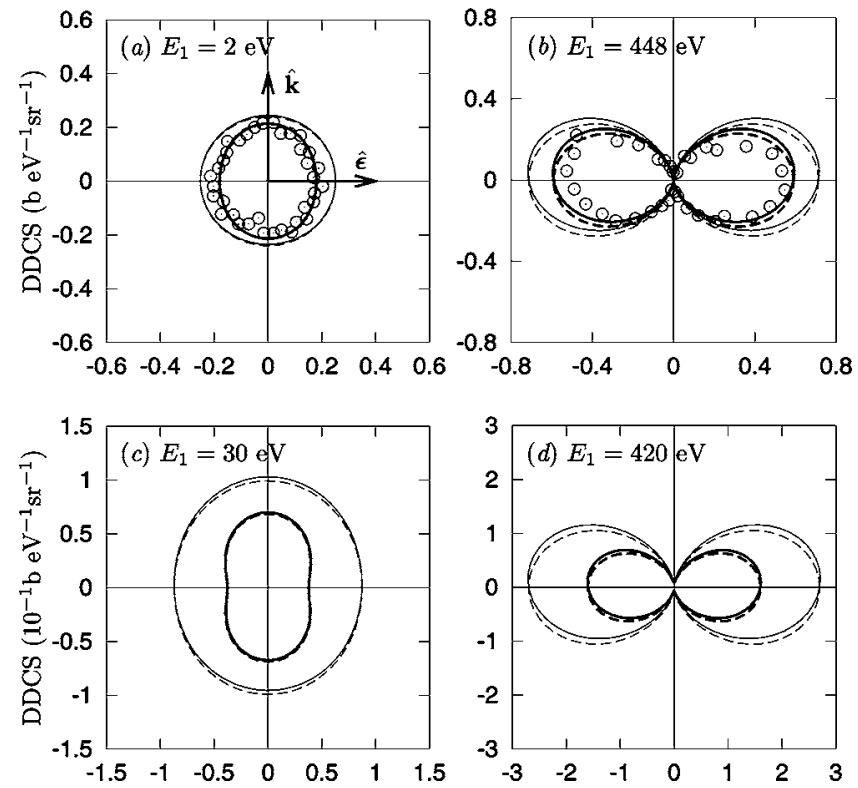

FIG. 2. DDCS for DPI of He for an excess energy of $450 \mathrm{eV}$, linear polarization, and coplanar geometry. Directions of the photon wave vector $\hat{\mathbf{k}}$ and polarization $\hat{\boldsymbol{\epsilon}}$ are as shown in (a). The angular distributions for the electron having energy $E_{1}$ are shown. Curves are defined as in Fig. 1. Experimental results [29] are normalized to the $\mathrm{CCC}$ results.

general conclusions on the magnitude of asymmetry effects in the DDCS. Similarly to the parameter $\beta^{S P I}$, the parameter $\beta$ for DPI must be within the range $-1 \leqslant \beta \leqslant 2$. One sees from Table I that this rule is satisfied; $\beta$ is close to 2 for the angular distribution of the faster electron and becomes small for the angular distribution of the slower electron. The
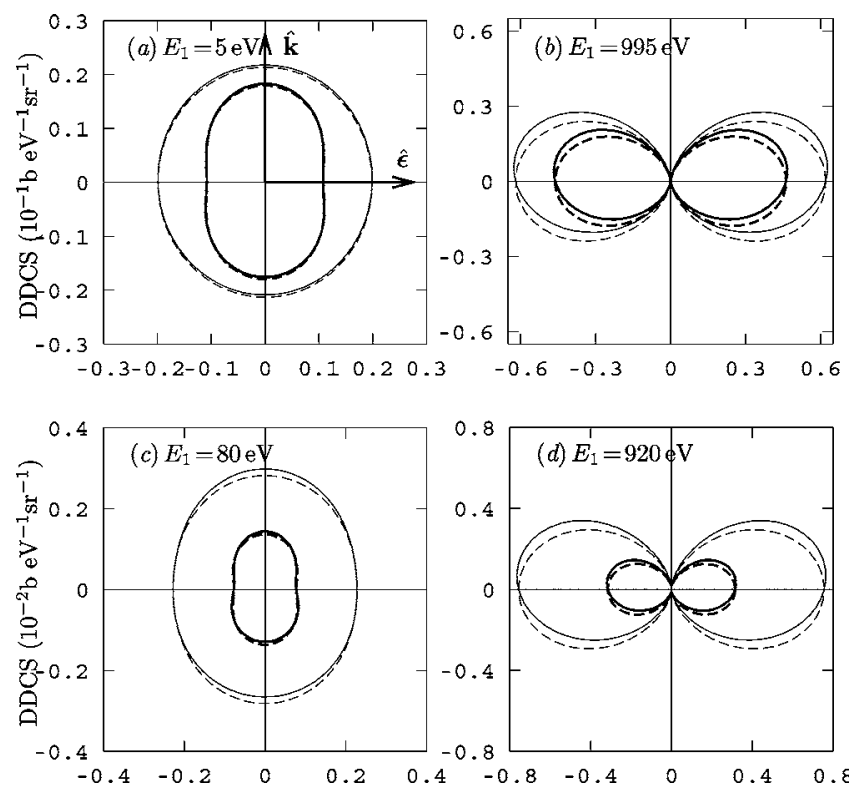

FIG. 3. DDCS for DPI of He for an excess energy of $1 \mathrm{keV}$, linear polarization, and coplanar geometry. Directions of the photon wave vector $\hat{\mathbf{k}}$ and polarization $\hat{\boldsymbol{\epsilon}}$ are as shown in (a). Angular distributions for the electron having energy $E_{1}$ are shown. Curves are defined as in Fig. 1.

dipole-quadrupole parameter $\gamma$ is large only for the angular distributions of the faster electron, but it always remains smaller than $\beta$. In contrast to SPI from atomic $s$ subshells, the dipole-quadrupole parameter $\delta$ in Table I is nonzero in all cases. For the angular distribution of the faster electron, it is found to be much smaller than $\gamma$, while for the angular distribution of the slower electron, $\gamma$ and $\delta$ are of comparable magnitude and in some cases $\delta$ is larger than $\gamma$. In general, the parameter $\gamma$ is positive and considerably less sensitive to the energy sharing than $\beta$ and $\gamma$.

Table I allows also for quantitative assessment of the accuracy of the LOPT results for the DDCS by comparing them to the corresponding results obtained by the CCC approach. As has already been found in Figs. 2-4, the LOPT approach generally overestimates the magnitude of the SDCS, $\sigma_{0}\left(E_{1}\right)$. One sees however, that for angular distributions of the faster electron (i.e., for $E_{1}>E_{\mathrm{exc}} / 2$ ), there is excellent agreement between the CCC and LOPT predictions for the asymmetry parameters $\beta, \gamma$, and $\delta$. This is because the definitions of these asymmetry parameters involve ratios of the dipole and quadrupole matrix elements and are thus, in general, less sensitive to the absolute quantitative accuracy of a particular model. However, for angular distributions of the slower electron (i.e., for $E_{1}<E_{\mathrm{exc}} / 2$ ), the level of agreement (or disagreement) between the CCC and LOPT predictions varies considerably. For angular distributions of the slower electron, there are also instances of disagreement between the parameters calculated by the CCC approach in the velocity gauge (not shown) and length gauge. This suggests that angular distributions of the slower electron are extremely sensitive to the accuracy within which the twoelectron reduced matrix elements are calculated.

\section{Singly differential cross sections}

In Fig. 4, we compare the LOPT results for the SDCS, $\sigma_{0}\left(E_{1}\right)$, for an excess energy of $450 \mathrm{eV}$, for which experimental measurements and CCC results were reported in Ref. [29]. For symmetric energy sharing, the LOPT results overestimate the SDCS by approximately a factor of 2.5 as compared to the CCC results. For strongly asymmetric sharing, the LOPT results agree with the CCC results. The magnitudes of the SDCS (given by the parameter $\sigma_{0}$ ) are presented in Table I for three excess energies and different energy sharings.

\section{B. Total cross sections for $\boldsymbol{K}$-shell double ionization of multielectron neutral atoms}

The two quantities that are usually measured in experiments on $K$-shell DPI of neutral atoms are the total $K$-shell double ionization cross section, $\sigma^{++}$, and the ratio of double to single ionization cross sections,

$$
R(\omega)=\frac{\sigma^{++}(\omega)}{\sigma^{+}(\omega)},
$$

where $\sigma^{+}(\omega)$ is the $K$-shell single-ionization cross section. The absolute total cross section $\sigma^{++}(\omega)$ and the ratio $R(\omega)$ for He were measured in the experiment of Ref. [15]. The ex- 
TABLE I. Photoelectron angular distribution parameters $\sigma_{0}, \beta, \gamma$, and $\delta$ evaluated according to Eqs. (24)-(27) by the CCC (length gauge) and LOPT approaches for three excess energies, $E_{\text {exc }}$, and various energy sharings.

\begin{tabular}{|c|c|c|c|c|c|c|c|c|c|}
\hline \multirow[b]{2}{*}{$E_{\mathrm{exc}}(\mathrm{eV})$} & \multirow[b]{2}{*}{$E_{1}(\mathrm{eV})$} & \multicolumn{4}{|c|}{$\mathrm{CCC}$} & \multicolumn{4}{|c|}{ LOPT } \\
\hline & & $\sigma_{0}(\mathrm{~b} / \mathrm{eV})$ & $\beta$ & $\gamma$ & $\delta$ & $\sigma_{0}(\mathrm{~b} / \mathrm{eV})$ & $\beta$ & $\gamma$ & $\delta$ \\
\hline \multirow[t]{2}{*}{100} & 99 & 99.9 & 1.44 & 0.13 & 0.015 & 118.3 & 1.57 & 0.14 & 0.012 \\
\hline & 1 & 99.9 & 0.28 & -0.007 & -0.0025 & 118.3 & 0.23 & -0.016 & 0.023 \\
\hline \multirow[t]{6}{*}{450} & 448 & 2.54 & 1.92 & 0.50 & 0.0055 & 3.06 & 1.93 & 0.51 & 0.0043 \\
\hline & 2 & 2.54 & -0.11 & 0.0040 & 0.0008 & 3.06 & 0.031 & -0.0054 & 0.020 \\
\hline & 420 & 0.73 & 1.74 & 0.43 & 0.013 & 1.19 & 1.83 & 0.46 & 0.011 \\
\hline & 30 & 0.73 & -0.35 & -0.009 & 0.021 & 1.19 & -0.084 & -0.019 & 0.039 \\
\hline & 400 & 0.44 & 1.58 & 0.36 & 0.022 & 0.76 & 1.74 & 0.42 & 0.016 \\
\hline & 50 & 0.44 & -0.34 & -0.050 & 0.031 & 0.76 & -0.061 & -0.038 & 0.049 \\
\hline \multirow[t]{4}{*}{1000} & 995 & 0.200 & 1.97 & 0.76 & 0.0030 & 0.262 & 1.98 & 0.77 & 0.0023 \\
\hline & 5 & 0.200 & -0.31 & -0.047 & 0.023 & 0.262 & -0.045 & -0.0006 & 0.021 \\
\hline & 920 & 0.015 & 1.70 & 0.71 & 0.016 & 0.033 & 1.86 & 0.73 & 0.013 \\
\hline & 80 & 0.015 & -0.34 & -0.15 & 0.062 & 0.033 & -0.14 & -0.066 & 0.062 \\
\hline
\end{tabular}

perimental measurements of the ratio $R(\omega)$ for $K$-shell DPI of several other neutral atoms were reported in Refs. [14,16-18].

From a theoretical perspective, the $K$-shell DPI process presents an additional challenge owing to the fact that $K$-shell electrons do not experience a purely Coulombic potential of the nucleus, even in the zero-order approximation in the interelectron interaction. Because in high- $Z$ atoms the $K$-shell electrons are localized near the nucleus while the outer electrons are relatively far from the nucleus, the screening effect of the outer electrons on the $K$-shell electrons may be approximated by using an effective charge, $Z_{\text {eff }}$, defined as $Z_{\text {eff }}=\sqrt{2 I^{\text {expt }}}$, where $I^{\text {expt }}$ is the value of the single-ionization potential for the $K$-shell [13]. Using this approximation as the basis for a LOPT account of electron correlations within the $K$-shell, Ref. [13] reports theoretical predictions for the ratio $R(\omega)$ for $\mathrm{Ne}, \mathrm{Ti}, \mathrm{Cr}, \mathrm{Fe}, \mathrm{Ni}, \mathrm{Co}$, and $\mathrm{Mo}$, most of which

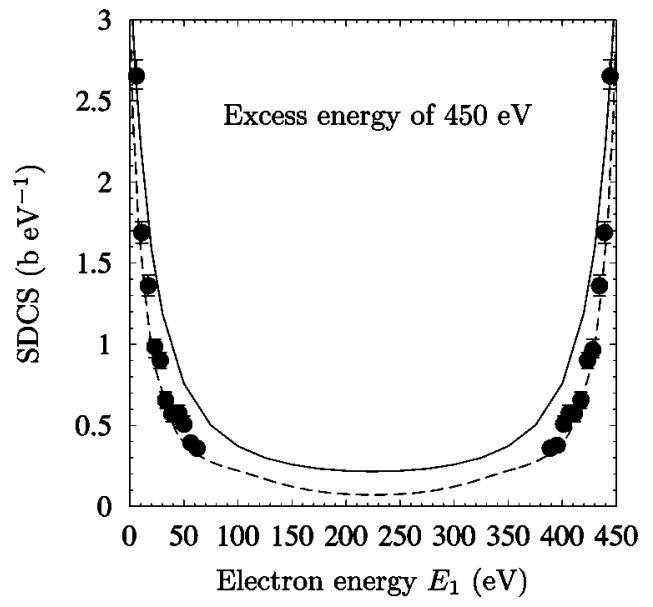

FIG. 4. SDCS for DPI of He at an excess energy of $450 \mathrm{eV}$. Full curve: present LOPT results; dashed curve: CCC results from Ref. [29]; the experimental data (from Ref. [29]) are normalized to the $\mathrm{CCC}$ results. are in excellent agreement with the experimental data in Refs. [14,16-18]. Significant discrepancies with experiment do exist for $\mathrm{Ni}$ (in which case the photon energy is very close to the double-ionization threshold) and for Mo (whose nuclear charge is rather high) [13].

Owing to the importance of $K$-shell DPI cross sections and branching ratios for multielectron neutral atoms, in the present work we revisit the calculations of Ref. [13] in an attempt to resolve the discrepancies mentioned above. To calculate a total cross section $\sigma^{+}(\omega)$ for $K$-shell single ionization, we use the independent-particle approximation, as in Ref. [13]. In this approximation, $\sigma^{+}(\omega)$ is equal to twice the hydrogen-like ion ionization cross section, $\sigma_{H}^{+}(\omega)$,

$$
\sigma_{H}^{+}(\omega)=\frac{2^{10}}{3} \pi^{2} \alpha \omega \frac{\eta^{10}}{Z^{4}\left(1+\eta^{2}\right)^{5}} \frac{e^{-4 \eta \arctan (1 / \eta)}}{1-e^{-2 \pi \eta}},
$$

where $\eta=Z / p$, where $p$ is the photoelectron momentum, and $Z=Z_{\text {eff }}$.

In Table II, we present our predictions for the absolute total DPI cross sections and for the ratios $R(\omega)$ for $\mathrm{He}$ and other multielectron atoms. For the case of He, whose $K$-shell is the only one, we use the bare nuclear charge, $Z=2$. Our predictions for He are compared with the experimental data in Ref. [15], while predictions for other atoms are compared with those in Ref. [13] and with the experimental data in Refs. [14,16-18]. For DPI of He, one sees that our results overestimate the total cross section (by about 46\%) and $R(\omega)$ (by about 10\%) as compared to the experimental results [15]. For atoms with higher $Z$, however, in most cases there is excellent agreement of our results for $R(\omega)$ with the experimental data as well as with the calculations in Ref. [13]. We note that for Mo, we confirm the result of Ref. [13], in spite of the fact that it disagrees with experiment [16]. For the case of $\mathrm{Ni}$, we have not been able to obtain a reliable prediction to be compared with that of Ref. [13] owing to the photon energy being close to the double-ionization threshold. Also, Mikhailov et al. [13] mention that for $\mathrm{Ni}$ there are large 
TABLE II. Total DPI cross section $\sigma^{++}$and ratio of double-ionization to single-ionization cross sections, $R=\sigma^{++} / \sigma^{+}$, for photon energy $\omega$ for double ionization from the $K$-shell of a neutral multielectron atom having nuclear charge $Z$ and experimental $K$-shell single-ionization potential $I^{\text {expt }}$. Present LOPT results are compared to predictions in Ref. [13] and experimental data where available.

\begin{tabular}{|c|c|c|c|c|c|c|c|c|c|c|}
\hline \multirow[b]{2}{*}{ Neutral Atom } & \multirow[b]{2}{*}{$\mathrm{Z}$} & \multirow[b]{2}{*}{$Z_{\text {eff }}$} & \multirow[b]{2}{*}{$I^{\text {expt }}(\mathrm{keV})$} & \multirow[b]{2}{*}{$\omega(\mathrm{keV})$} & \multicolumn{2}{|c|}{$\sigma^{++}(\omega)(b)$} & \multicolumn{4}{|c|}{$R(\omega)=\sigma^{++}(\omega) / \sigma^{+}(\omega)$} \\
\hline & & & & & LOPT & Exp. [15] & LOPT & Theory [13] & Exp. & Ref. \\
\hline $\mathrm{He}$ & 2 & 2.0 & 0.0246 & 0.53 & 126.0 & $86.4^{\mathrm{a}}$ & $0.31 \times 10^{-1}$ & & $0.282 \times 10^{-1 b}$ & [15] \\
\hline $\mathrm{Ne}$ & 10 & 8.0 & 0.87 & 5 & 3.63 & & $0.28 \times 10^{-2}$ & $0.28 \times 10^{-2}$ & $0.32(4) \times 10^{-2}$ & [18] \\
\hline $\mathrm{Ti}$ & 22 & 19.11 & 4.97 & 17.4 & 0.51 & & $0.51 \times 10^{-3}$ & $0.51 \times 10^{-3}$ & $0.53 \times 10^{-3}$ & [14] \\
\hline $\mathrm{Cr}$ & 24 & 20.98 & 5.99 & 17.4 & 0.53 & & $0.37 \times 10^{-3}$ & $0.37 \times 10^{-3}$ & $0.38 \times 10^{-3}$ & [14] \\
\hline $\mathrm{Fe}$ & 26 & 22.88 & 7.12 & 17.4 & 0.45 & & $0.224 \times 10^{-3}$ & $0.23 \times 10^{-3}$ & $0.24 \times 10^{-3}$ & [14] \\
\hline $\mathrm{Ni}$ & 28 & 24.76 & 8.34 & 17.4 & & & & $0.51 \times 10^{-4}$ & $1.1 \times 10^{-4}$ & [14] \\
\hline $\mathrm{Cu}$ & 29 & 25.70 & 8.99 & 20 & 0.24 & & $1.13 \times 10^{-4}$ & $1.1 \times 10^{-4}$ & $1.3(3) \times 10^{-4}$ & [17] \\
\hline Mo & 42 & 38.35 & 20.01 & 50 & 0.058 & & $0.87 \times 10^{-4}$ & $0.87 \times 10^{-4}$ & $3.4(6) \times 10^{-4}$ & [16] \\
\hline
\end{tabular}

${ }^{\mathrm{a}}$ This value corresponds to $\omega=0.50 \mathrm{keV}[15]$.

${ }^{\mathrm{b}}$ This value corresponds to $\omega=0.52 \mathrm{keV}[15]$.

uncertainties in both their prediction and the result of the experimental measurement [14].

\section{CONCLUSIONS}

We have derived two alternative, model-independent representations for the dipole and quadrupole angular distribution asymmetry parameters for the DDCS of DPI from a ${ }^{1} S_{0}$ state: (i) in terms of the polarization-invariant amplitudes [cf. Eqs. (18)-(21)] and (ii) in terms of the exact two-electron reduced matrix elements [cf. Eqs. (24)-(27)]. The first parametrization may be most suitable for analyzing experimental data (e.g., when polarization-invariant amplitudes are extracted from the TDCS measurements), while the second parametrization may be most suitable for theoretical calculations (when the reduced matrix elements are evaluated within a particular dynamical model). We have found that in contrast to SPI, for which the dipole-quadrupole parameter $\delta^{S P I}$ vanishes for ionization from initial atomic $s$ subshells, the parameter $\delta$ for DPI from a ${ }^{1} S_{0}$ state is generally nonzero. Therefore, the parametrizations for the dipolequadrupole TDCS in Ref. [8], the parametrizations for the DDCS in Eqs. (24)-(27), and either the set of reduced matrix elements [defined in Eq. (11)] or the dynamical factors [defined in Eq. (16)] permit one to reconstruct the dipolequadrupole TDCS and DDCS for any experimental geometry and for any polarization state of a photon beam. We note that the reduced matrix elements or dynamical factors are the only quantities that should be calculated numerically for a given photon frequency and excess energy sharing within a particular dynamical model of DPI.

We have used the parametrizations in Eqs. (24)-(27) to calculate DDCSs and SDCSs within the CCC and LOPT approaches. Our results for the DDCS account for the lowest-order nondipole effects (described by the asymmetry parameters $\gamma$ and $\delta$ ) and exhibit forward-backward asymmetries, which become very significant for an excess energy of $1 \mathrm{keV}$. The CCC results presented here thus provide the first accurate numerical predictions for the magnitude of nondi- pole effects in angle-integrated DPI cross sections. We find good agreement between CCC and LOPT results for the asymmetry parameters $\beta, \gamma$, and $\delta$ for angular distributions of the faster electron; for angular distributions of the slower electron (in which case the asymmetry parameters are much smaller), the level of agreement (or disagreement) of the two results varies from case to case.

We have also used our parametrization for $\sigma_{0}$ together with the LOPT approach to calculate total DPI cross sections and ratios of double-ionization to single-ionization cross sections for double ejection from the $K$-shell of $\mathrm{He}$ and other multielectron neutral atoms. For the case of the He atom, our results generally overestimate the absolute experimental data, as would be expected for a perturbative treatment. For atoms with higher $Z$ (i.e., when electron correlations are less important) our predictions are in excellent agreement with the results of other theoretical calculations and with experimental measurements, except for the case when the photon energy is close to the double-ionization threshold.

\section{ACKNOWLEDGMENTS}

This work was supported in part by the U.S. Department of Energy, Office of Science, Division of Chemical Sciences, Geosciences, and Biosciences, under Grant No. DE-FG0396ER14646, by the Russian Foundation for Basic Research, under Grant No. 04-02-16350, and by the joint Grant No. VZ-010-0 of the Civilian Research and Development Foundation and the RF Ministry of Education (N. L. M. and A. V. M.). The LOPT and CCC computations presented in this paper were performed utilizing the Research Computing Facility of the University of Nebraska-Lincoln and the Compaq AlphaServer SC National Facility of the Australian Partnership for Advanced Computing.

\section{APPENDIX A: EVALUATION OF INTEGRALS WITH SCALAR PRODUCTS}

Here we evaluate the three kinds of integrals involving scalar products that appear in Eqs. (17). We shall assume that 
the $z$ axis of the coordinate frame for the vector $\hat{\mathbf{p}}_{2}$ is directed along the vector $\hat{\mathbf{p}}_{1}$.

The integration of the term $\left(\mathbf{a} \cdot \hat{\mathbf{p}}_{2}\right) u_{1}(\cos \theta)$ is trivial and yields the following result:

$$
\int d \Omega_{\mathbf{p}_{2}}\left(\mathbf{a} \cdot \hat{\mathbf{p}}_{2}\right) u_{1}(\cos \theta)=2 \pi\left(\mathbf{a} \cdot \hat{\mathbf{p}}_{1}\right) \int_{-1}^{1} x u_{1}(x) d x,
$$

where we have used the fact that $a_{z}=\left(\mathbf{a} \cdot \hat{\mathbf{p}}_{1}\right)$.

The integral of the term $\left(\mathbf{a} \cdot \hat{\mathbf{p}}_{2}\right)\left(\mathbf{b} \cdot \hat{\mathbf{p}}_{2}\right) u_{2}(\cos \theta)$ can be evaluated by employing the tensor decomposition [24]

$$
\begin{aligned}
\left(\mathbf{a} \cdot \hat{\mathbf{p}}_{2}\right)\left(\mathbf{b} \cdot \hat{\mathbf{p}}_{2}\right) & =\sum_{k=0}^{2}(-1)^{k}\left(\{\mathbf{a} \otimes \mathbf{b}\}_{k} \cdot\left\{\hat{\mathbf{p}}_{2} \otimes \hat{\mathbf{p}}_{2}\right\}_{k}\right) \\
& =\frac{1}{3}(\mathbf{a} \cdot \mathbf{b})+\sqrt{\frac{2}{3}}\left(\{\mathbf{a} \otimes \mathbf{b}\}_{2} \cdot C_{2}\left(\theta, \phi_{2}\right)\right),
\end{aligned}
$$

where $C_{2 \mu}\left(\theta, \phi_{2}\right)$ is the modified spherical harmonic. By noting that

$$
\int_{0}^{2 \pi} C_{2 \mu}\left(\theta, \phi_{2}\right) d \phi_{2}=2 \pi P_{2}(\cos \theta) \delta_{\mu, 0}
$$

and

$$
\begin{aligned}
\{\mathbf{a} \otimes \mathbf{b}\}_{20} & =\left(\{\mathbf{a} \otimes \mathbf{b}\}_{2} \cdot C_{2}\left(\hat{\mathbf{p}}_{1}\right)\right) \\
& =\sqrt{\frac{3}{2}}\left(\left(\mathbf{a} \cdot \hat{\mathbf{p}}_{1}\right)\left(\mathbf{a} \cdot \hat{\mathbf{p}}_{1}\right)-\frac{1}{3}(\mathbf{a} \cdot \mathbf{b})\right),
\end{aligned}
$$

we obtain the following result:

$$
\begin{aligned}
\int d \Omega_{\mathbf{p}_{2}}\left(\mathbf{a} \cdot \hat{\mathbf{p}}_{2}\right)\left(\mathbf{b} \cdot \hat{\mathbf{p}}_{2}\right) u_{2}(\cos \theta) \\
=\frac{2 \pi}{3}(\mathbf{a} \cdot \mathbf{b}) \int_{-1}^{1} u_{2}(x)\left(1-P_{2}(x)\right) d x \\
\quad+2 \pi\left(\mathbf{a} \cdot \hat{\mathbf{p}}_{1}\right)\left(\mathbf{b} \cdot \hat{\mathbf{p}}_{1}\right) \int_{-1}^{1} u_{2}(x) P_{2}(x) d x .
\end{aligned}
$$

The integral of the term $\left(\mathbf{a} \cdot \hat{\mathbf{p}}_{2}\right)\left(\mathbf{b} \cdot \hat{\mathbf{p}}_{2}\right)\left(\mathbf{c} \cdot \hat{\mathbf{p}}_{2}\right) u_{3}(\cos \theta)$ can be evaluated similarly using the tensor decomposition

$$
\begin{aligned}
(\mathbf{a} \cdot & \left.\hat{\mathbf{p}}_{2}\right)\left(\mathbf{b} \cdot \hat{\mathbf{p}}_{2}\right)\left(\mathbf{c} \cdot \hat{\mathbf{p}}_{2}\right) \\
\quad & =\sum_{k=0}^{2} \sum_{q=|k-1|}^{k+1}\left(\left\{\{\mathbf{a} \otimes \mathbf{b}\}_{k} \otimes \mathbf{c}\right\}_{q} \cdot\left\{\left\{\hat{\mathbf{p}}_{2} \otimes \hat{\mathbf{p}}_{2}\right\}_{k} \otimes \hat{\mathbf{p}}_{2}\right\}_{q}\right) \\
& =\frac{1}{5}\left[\left(\mathbf{a} \cdot \hat{\mathbf{p}}_{2}\right)(\mathbf{b} \cdot \mathbf{c})+\left(\mathbf{b} \cdot \hat{\mathbf{p}}_{2}\right)(\mathbf{a} \cdot \mathbf{c})+\left(\mathbf{c} \cdot \hat{\mathbf{p}}_{2}\right)(\mathbf{a} \cdot \mathbf{b})\right] \\
& +\sqrt{\frac{2}{5}}\left(\left\{\{\mathbf{a} \otimes \mathbf{b}\}_{2} \otimes \mathbf{c}\right\}_{3} \cdot C_{3}\left(\theta, \phi_{2}\right)\right) .
\end{aligned}
$$

The integral of the first term (involving a single scalar product) in this equation is evaluated according to Eq. (A1). The integration of the second term [involving $C_{3}\left(\theta, \phi_{2}\right)$ ] over $\phi_{2}$ yields the scalar product $\left(\left\{\{\mathbf{a} \otimes \mathbf{b}\}_{2} \otimes \mathbf{c}\right\}_{3} \cdot\left\{\hat{\mathbf{p}}_{1}\right\}_{3}\right)$ (where $\{\hat{\mathbf{p}}\}_{3}$ $\left.\equiv\left\{\{\hat{\mathbf{p}} \otimes \hat{\mathbf{p}}\}_{2} \otimes \hat{\mathbf{p}}\right\}_{3}\right)$. The expression for this scalar product may be obtained from Eq. (A6) by making substitutions $\left(\hat{\mathbf{p}}_{2}, \varphi_{2}\right) \rightarrow\left(\hat{\mathbf{p}}_{1}, \varphi_{1}\right)$ and by noting that $C_{3}\left(\theta, \phi_{1}\right)$ $=\sqrt{(5 / 2)}\left\{\hat{\mathbf{p}}_{1}\right\}_{3}$. One finds therefore,

$$
\begin{aligned}
\left(\left\{\{\mathbf{a} \otimes \mathbf{b}\}_{2} \otimes \mathbf{c}\right\}_{3} \cdot\left\{\hat{\mathbf{p}}_{1}\right\}_{3}\right)= & \left(\mathbf{a} \cdot \hat{\mathbf{p}}_{1}\right)\left(\mathbf{b} \cdot \hat{\mathbf{p}}_{1}\right)\left(\mathbf{b} \cdot \hat{\mathbf{p}}_{1}\right)-\frac{1}{5}\left[\left(\mathbf{a} \cdot \hat{\mathbf{p}}_{1}\right)\right. \\
& \times(\mathbf{b} \cdot \mathbf{c})+\left(\mathbf{b} \cdot \hat{\mathbf{p}}_{1}\right)(\mathbf{a} \cdot \mathbf{c}) \\
& \left.+\left(\mathbf{c} \cdot \hat{\mathbf{p}}_{1}\right)(\mathbf{a} \cdot \mathbf{b})\right] .
\end{aligned}
$$

Consequently, we arrive at the following expression:

$$
\begin{aligned}
\int d \Omega_{\mathbf{p}_{2}}\left(\mathbf{a} \cdot \hat{\mathbf{p}}_{2}\right)\left(\mathbf{b} \cdot \hat{\mathbf{p}}_{2}\right)\left(\mathbf{c} \cdot \hat{\mathbf{p}}_{2}\right) u_{3}(\cos \theta) \\
=2 \pi\left(\mathbf{a} \cdot \hat{\mathbf{p}}_{1}\right)\left(\mathbf{b} \cdot \hat{\mathbf{p}}_{1}\right)\left(\mathbf{c} \cdot \hat{\mathbf{p}}_{1}\right) \int_{-1}^{1} u_{3}(x) P_{3}(x) d x \\
\quad+\frac{2 \pi}{5}\left[\left(\mathbf{a} \cdot \hat{\mathbf{p}}_{1}\right)(\mathbf{b} \cdot \mathbf{c})+\left(\mathbf{b} \cdot \hat{\mathbf{p}}_{1}\right)(\mathbf{a} \cdot \mathbf{c})+\left(\mathbf{c} \cdot \hat{\mathbf{p}}_{1}\right)(\mathbf{a} \cdot \mathbf{b})\right] \\
\quad \times \int_{-1}^{1} u_{3}(x)\left[x-P_{3}(x)\right] d x .
\end{aligned}
$$

\section{APPENDIX B: EVALUATION OF THE DPI QUADRUPOLE MATRIX ELEMENTS BY THE CCC APPROACH}

The reduced matrix elements, $d_{l_{1} l_{2}}\left(p_{1}, p_{2}\right)$ and $q_{l_{1} l_{2}}\left(p_{1}, p_{2}\right)$, of the dipole and quadrupole electromagnetic interaction operators that appear in Eqs. (24)-(27) have been evaluated as the limit $q \rightarrow 0$ of the matrix elements of the Born operator $e^{i \mathbf{q} \cdot \mathbf{r}}$, which have been calculated within the CCC approach for study of the $(e, 3 e)$ process in $\mathrm{He}$ [26]. For a geometry in which the vector $\mathbf{q}$ is directed along the $z$ axis, the matrix elements that have been evaluated numerically by the CCC approach have the following form:

$$
\begin{aligned}
\left\langle\psi_{\mathbf{p}_{1} \mathbf{p}_{2}}\left|e^{i q z_{1}}+e^{i q z_{2}}\right| \psi_{E_{0}}\right\rangle= & -\frac{1}{\sqrt{3}} \sum_{J=0}^{\infty} \sum_{l_{1} m_{1} l_{2} m_{2}} i^{J-l_{1}-l_{2}} \\
& \times \Pi_{J}^{2} C_{l_{1} m_{1} l_{2} m_{2}}^{10} Y_{l_{1} m_{1}}\left(\hat{\mathbf{p}}_{1}\right) Y_{l_{2} m_{2}}\left(\hat{\mathbf{p}}_{2}\right) \\
& \times B_{l_{1} l_{2} J}\left(p_{1}, p_{2} ; q\right),
\end{aligned}
$$

where $B_{l_{1} l_{2} J}\left(p_{1}, p_{2} ; q\right)$ is the product of the $T$-matrix integrated Born matrix element (including Coulomb phases) and the projection of the Coulomb wave onto the matching energy pseudostate (see Ref. [26] for details); $\Pi_{a b \ldots c}$ $\equiv \sqrt{(2 a+1)(2 b+1) \cdots(2 c+1)}$. The quantity $B_{l_{1} l_{2} J}\left(p_{1}, p_{2} ; q\right)$ can be calculated by the existing $(e, 3 e) \mathrm{CCC}$ code. Therefore, the goal here is to relate this quantity to the DPI reduced matrix elements, $d_{l l^{\prime}}\left(p_{1}, p_{2}\right)$ and $q_{l l^{\prime}}\left(p_{1}, p_{2}\right)$ (calculated using the length form of the dipole and quadrupole electromagnetic interaction operators).

First, for an arbitrarily directed vector $\mathbf{q}$, one obtains 


$$
\begin{aligned}
& \left\langle\psi_{\mathbf{p}_{1} \mathbf{p}_{2}}\left|e^{i \mathbf{q} \cdot \mathbf{r}_{1}}+e^{i \mathbf{q} \cdot \mathbf{r}_{2}}\right| \psi_{E_{0}}\right\rangle \\
& =\sqrt{\frac{4 \pi}{3}} \sum_{J=0}^{\infty} \sum_{M=-J}^{J}(-1)^{M+1} \\
& \quad \times \Pi_{J} Y_{J M}(\hat{\mathbf{q}}) \sum_{l_{1} m_{1}} \sum_{l_{2} m_{2}} i^{J-l_{1}-l_{2}} C_{l_{1} m_{1} l_{2} m_{2}}^{1-M} \\
& \quad \times Y_{l_{1} m_{1}}\left(\hat{\mathbf{p}}_{1}\right) Y_{l_{2} m_{2}}\left(\hat{\mathbf{p}}_{2}\right) B_{l_{1} l_{2} J}\left(p_{1}, p_{2} ; q\right) .
\end{aligned}
$$

Second, using the standard multipole expansion,

$$
e^{i \mathbf{q} \cdot \mathbf{r}}=4 \pi \sum_{J=0}^{\infty} i^{J} j_{J}(q r) \sum_{M=-J}^{J} Y_{J M}^{*}(\hat{\mathbf{q}}) Y_{J M}(\hat{\mathbf{r}}),
$$

and the asymptotic formula

$$
j_{J}(q r) \rightarrow \frac{(q r)^{J}}{(2 J+1) ! !} \text { as } q \rightarrow 0,
$$

in the left-hand side of Eq. (B1), one obtains the following relation:

$$
\begin{aligned}
\left\langle\psi_{\mathbf{p}_{1} \mathbf{p}_{2}}\right. & r_{1}^{J} Y_{J M}\left(\hat{\mathbf{r}}_{1}\right)+r_{2}^{J} Y_{J M}\left(\hat{\mathbf{r}}_{2}\right)\left|\psi_{E_{0}}\right\rangle \\
= & (-1)^{J} \frac{(2 J+1) ! !}{(4 \pi)^{3 / 2}} \sum_{l_{1}, l_{2}} i^{-\left(l_{1}+l_{2}\right)} \Pi_{l_{1} l_{2}} \mathcal{C}_{J M}^{l_{1} l_{2}}\left(\hat{\mathbf{p}}_{1}, \hat{\mathbf{p}}_{2}\right) \\
& \times \lim _{q \rightarrow 0}\left[q^{-J} B_{l_{1} l_{2} J}\left(p_{1}, p_{2} ; q\right)\right],
\end{aligned}
$$

where $\mathcal{C}_{J M}^{l_{1} l_{2}}\left(\hat{\mathbf{p}}_{1}, \hat{\mathbf{p}}_{2}\right)$ is a modified bipolar harmonic. By means of Eq. (B4), it is now possible to obtain the desired relations between length-form matrix elements $d_{l l^{\prime}}$ and $q_{l l^{\prime}}$ and the quantity $B_{l l^{\prime} J}\left(p_{1}, p_{2} ; q\right)$ for $q \ll 1$. By rewriting the dipole and quadrupole operators as

$$
\begin{gathered}
\mathbf{D}=i \omega \sqrt{\frac{4 \pi}{3}}\left[r_{1} Y_{1}\left(\hat{\mathbf{r}}_{1}\right)+r_{2} Y_{1}\left(\hat{\mathbf{r}}_{2}\right)\right], \\
Q_{2}=-\frac{1}{2} \alpha \omega^{2} \sqrt{\frac{8 \pi}{15}}\left[r_{1}^{2} Y_{2}\left(\hat{\mathbf{r}}_{1}\right)+r_{2}^{2} Y_{2}\left(\hat{\mathbf{r}}_{2}\right)\right],
\end{gathered}
$$

and comparing Eq. (B4) for $J=1,2$ and Eqs. (B5) and (B6) to Eqs. (9)-(11), one obtains the following:

$$
\begin{gathered}
d_{l_{1} l_{2}}\left(p_{1}, p_{2}\right)=3 i^{l_{1}+l_{2}+1} \frac{\Pi_{l_{1} l_{2}}}{4 \pi} \omega \lim _{q \rightarrow 0} \frac{B_{l_{1} l_{2} 1}\left(p_{1}, p_{2} ; q\right)}{q}, \\
q_{l_{1} l_{2}}\left(p_{1}, p_{2}\right)=5 i^{l_{1}+l_{2}} \sqrt{\frac{3}{2}} \frac{\Pi_{l_{1} l_{2}}}{4 \pi} \alpha \omega^{2} \lim _{q \rightarrow 0} \frac{B_{l_{1} l_{2} 2}\left(p_{1}, p_{2} ; q\right)}{q^{2}} .
\end{gathered}
$$

In numerical calculations, the value of $q=0.01$ has been used. It has been shown that the dipole matrix elements calculated according to Eq. (B7) agree well with those calculated using the CCC code for DPI. It has also been checked that the variation of the value of $q$ near $q=0.01$ does not change the results within several significant figures.
[1] J. S. Briggs and V. Schmidt, J. Phys. B 33, R1 (2000).

[2] G. C. King and L. Avaldi, J. Phys. B 33, R215 (2000).

[3] L. Avaldi and A. Huetz, J. Phys. B 38, S861 (2005).

[4] N. L. Manakov, S. I. Marmo, and A. V. Meremianin, J. Phys. B 29, 2711 (1996).

[5] L. Malegat, P. Selles, and A. Huetz, J. Phys. B 30, 251 (1997).

[6] A. Y. Istomin, N. L. Manakov, A. V. Meremianin, and A. F. Starace, Phys. Rev. Lett. 92, 063002 (2004).

[7] A. Y. Istomin, N. L. Manakov, A. V. Meremianin, and A. F. Starace, Phys. Rev. A 70, 010702(R) (2004).

[8] A. Y. Istomin, N. L. Manakov, A. V. Meremianin, and A. F. Starace, Phys. Rev. A 71, 052702 (2005).

[9] M. Ya. Amusia, E. G. Drukarev, E. G. Gorshkov, and M. P. Kazachkov, J. Phys. B 8, 1248 (1975).

[10] M. A. Kornberg and J. E. Miraglia, Phys. Rev. A 52, 2915 (1995).

[11] A. S. Kheifets and I. Bray, Phys. Rev. A 57, 2590 (1998).

[12] A. I. Mikhailov, I. A. Mikhailov, A. N. Moskalev, A. V. Nefiodov, G. Plunien, and G. Soff, Phys. Lett. A 316, 395 (2003).

[13] A. I. Mikhailov, I. A. Mikhailov, A. N. Moskalev, A. V. Nefiodov, G. Plunien, and G. Soff, Phys. Rev. A 69, 032703 (2004).

[14] J. Ahopelto, E. Rantavuori, and O. Keski-Rahkonen, Phys. Scr. 20, 71 (1979).

[15] J. A. R. Samson, W. C. Stolte, Z. X. He, J. N. Cutler, Y. Lu, and R. J. Bartlett, Phys. Rev. A 57, 1906 (1998).
[16] E. P. Kanter, R. W. Dunford, B. Krässig, and S. H. Southworth, Phys. Rev. Lett. 83, 508 (1999).

[17] R. Diamant, S. Huotari, K. Hämäläinen, C. C. Kao, and M. Deutsch, Phys. Rev. A 62, 052519 (2000).

[18] S. H. Southworth, E. P. Kanter, B. Krässig, L. Young, G. B. Armen, J. C. Levin, D. L. Ederer, and M. H. Chen, Phys. Rev. A 67, 062712 (2003).

[19] The analytic expressions for the dipole asymmetry parameters are given in Ref. [4] for arbitrary angular momentum of the initial and final atomic states and are thus presented in terms of multiple sums involving combinations of Clebsch-Gordon coefficients, and $6 j$ and $9 j$ symbols.

[20] A. S. Kheifets and I. Bray, J. Phys. B 31, L447 (1998).

[21] A. Knapp, A. Kheifets, I. Bray, Th. Weber, A. L. Landers, S. Schössler, T. Jahnke, J. Nickles, S. Kammer, O. Jagutzki, L. Ph. H. Schmidt, M. Schöffler, T. Osipov, M. H. Prior, H. Schmidt-Böcking, C. L. Cocke, and R. Dörner, J. Phys. B 38, 615, 634 (2005).

[22] A. Y. Istomin, N. L. Manakov, and A. F. Starace, J. Phys. B 35, L543 (2002).

[23] A. Y. Istomin, N. L. Manakov, and A. F. Starace, Phys. Rev. A 69, 032713 (2004).

[24] D. A. Varshalovich, A. N. Moskalev, and V. K. Khersonskii, Quantum Theory of Angular Momentum (World Scientific, Singapore, 1988).

[25] J. W. Cooper, Phys. Rev. A 47, 1841 (1993). 
[26] A. S. Kheifets, I. Bray, A. Duguet, A. Lahmam-Bennani, and I. Taouil, J. Phys. B 32, 5047 (1999).

[27] A. Knapp, M. Walter, Th. Weber, A. L. Landers, S. Schössler, T. Jahnke, M. Schöffler, J. Nickles, S. Kammer, O. Jagutzki, L. $\mathrm{Ph}$. H. Schmidt, T. Osipov, J. Rösch, M. H. Prior, H. SchmidtBöcking, C. L. Cocke, J. Feagin, and R. Dörner, J. Phys. B 35, L521 (2002).
[28] J. Colgan and M. S. Pindzola, J. Phys. B 37, 1153 (2004).

[29] A. Knapp, A. Kheifets, I. Bray, T. Weber, A. L. Landers, S. Schössler, T. Jahnke, J. Nickles, S. Kammer, O. Jagutzki, L. P. Schmidt, T. Osipov, J. Rösch, M. H. Prior, H. SchmidtBöcking, C. L. Cocke, and R. Dörner, Phys. Rev. Lett. 89, 033004 (2002). 